\title{
The Additive Weibull-Geometric Distribution: Theory and Applications
}

\author{
I. Elbatal \\ Department of Mathematics and Statistics - College of Science \\ Al Imam Mohammad Ibn Saud Islamic University- Saudi Arabia \\ iielbatal@imamu.edu.sa \\ M. M. Mansour \\ Department of Statistics, Mathematics and Insurance, Benha University, Egypt \\ Mahmoud.mansour@fcom.bu.edu.eg \\ Mohammad Ahsanullah \\ Department of Management Sciences, Rider University \\ Lawrenceville, NJ 08648-3009 \\ ahsan@rider.edu
}

Received 27 March 2014

Accepted 21 June 2015

\begin{abstract}
In this paper, we introduce a new class of lifetime distributions which is called the additive Weibull geometric (AWG) distribution. This distribution obtained by compounding the additive Weibull and geometric distributions. The new distribution has a number of well-known lifetime special sub-models such as modified Weibull geometric, Weibull geometric, exponential geometric, among several others. Some structural properties of the proposed new distribution are discussed. We propose the method of maximum likelihood for estimating the model parameters and obtain the observed information matrix. A real data set is used to illustrate the importance and flexibility of the new distribution.
\end{abstract}

Keywords: Additive Weibull distribution, Geometric distribution, Moments, Maximum likelihood.

2000 Mathematics Subject Classification: 60-XX, 60EXX.

\section{Introduction}

The study of life length of organisms, structures, materials, etc., is very important in the biological and engineering sciences. A substantial part of such study is devoted to modeling the lifetime data by a failure distribution. The exponential, Rayleigh, and Weibull distributions are the most commonly used distributions in reliability and life testing. These distributions have several desirable properties and nice physical interpretations. Unfortunately, however, the exponential and Rayleigh distributions have constant and increasing failure rates, respectively. The Weibull distribution generalizes both these distributions which may have increasing, constant, or decreasing failure rates. The modified Weibull distribution (Lai et al. (2003)) attracts some interest among researchers and practitioners because of its ability in modeling bathtub shaped failure rate data. Also, Sarhan and Zaindin (2009) introduced the modified Weibull distribution. Xie and Lai 
(1995) introduced a four-parameter distribution called the additive Weibull distribution based on the simple idea of combining the hazard rates of two Weibull distributions: one has a decreasing hazard rate and the other one has an increasing hazard rate.

The Weibull distribution, having exponential and Rayleigh as special cases, is a very popular distribution for modeling lifetime data and for modeling phenomenon with monotone failure rates. When modeling monotone hazard rates, the Weibull distribution may be an initial choice because of its negatively and positively skewed density shapes. However, the Weibull distribution does not provide a reasonable parametric fit for modeling phenomenon with non-monotone failure rates such as the bathtub-shaped and the unimodal failure rates which are common in reliability and biological studies. Such bathtub hazard curves have nearly flat middle portions and the corresponding densities have a positive anti-mode. An example of bathtub-shaped failure rate is the human mortality experience with a high infant mortality rate which reduces rapidly to reach a low. It then remains at that level for quite a few years before picking up again. Unimodal failure rates can be observed in course of a disease whose mortality reaches a peak after some finite period and then declines gradually.

In this article, we propose a new lifetime distribution, referred to as the additive Weibull geometric $(A W G)$ distribution which contains as special sub-models and study some of its properties. Several distributions have been proposed in the literature to model lifetime data by compounding some useful lifetime distributions. Adamidis and Loukas (1998) introduced a two-parameter exponential-geometric $(E G)$ distribution by compounding an exponential distribution with a geometric distribution. In the same way, the exponential Poisson $(E P)$ and exponential logarithmic (EL) distributions were introduced and studied by Kus (2007) and Tahmasbi and Rezaei (2008), respectively. Recently, Chahkandi and Ganjali (2009) proposed the exponential power series $(E P S)$ family of distributions, which contains as special cases these distributions. Barreto-Souza et al. (2010) introduced the Weibull-geometric (WG). Mahmoudi and Shiran (2012) presented exponentiated Weibull-Geometric distribution and its Applications. Nadarajah et al. (2014) introduced A new fourparameter lifetime distribution (generalized linear failure rate geometric distribution). Wang and Elbatal, (2014) proposed modified Weibull geometric distribution. (Adamidis et al. (2005) proposed the extended exponential-geometric $(E E G)$ distribution which generalizes the EG distribution and discussed various of its statistical properties along with its reliability features. The hazard function of the EEG distribution can be monotone decreasing, increasing or constant.

The paper is organized as follows. In Section 2, we define the $A W G$ distribution, its probability density function ( $p d f)$, cumulative distribution function $(c d f)$ and special cases of the $A W G$ distribution. In Section 3 , some properties of the new distribution are given. Residual life and reversed residual functions of the $A W G$ distribution, Bonferroni and Lorenz Curves and mean deviations are discussed in Section 4. In section 5. we demonstrate the maximum likelihood estimates of the unknown parameters. Finally, In section 6 we present a data analysis to illustrate the usefulness of the proposed distribution.

\section{A New Class}

Our class can be derived as follows. Suppose that a company has $N$ systems functioning independently and producing a certain product at a given time, where $N$ is a random variable, which is often determined by economy, customers demand, etc. The reason for considering $N$ as a random variable comes from a practical viewpoint in which failure (of a device for example) often occurs due to the present of an unknown number of initial defects in the system. In this paper, we focus on the case in which $N$ is discrete random variable following a geometric distribution (truncated at zero) with the probability mass function given by

$$
P(n ; p)=P(N=n)=(1-p) p^{\mathrm{n}-1}, \text { for } n \in N \text { and } p \in(0,1) \text {. }
$$

Note that $N$ can also be taken to follow other discrete distributions, such as binomial, Poisson, logarithmic, etc, whereas they need to be truncated zero because one must have $N \geq 1$. Now, let $X_{1}, X_{2}, \ldots, X_{N}$ be $N$ independent and identically distributed (iid) random variables from additive Weibull distribution with cumulative distribution function(cdf) is given by

$$
F(x)=1-e^{-\left(\theta x^{\alpha}+\beta x^{\lambda}\right),} x \geq 0
$$


where $\theta>0, \beta>0$ and $\alpha>\lambda>0$,or $\lambda>\alpha>0$ which gives identifiability to the model. When $\alpha>0$ the hazard rate is increasing and when $0<\lambda<1$ hazard rate is decreasing. The corresponding probability density function is

$$
f(x, \theta, \alpha, \beta, \lambda)=\left(\theta \alpha x^{\alpha-1}+\beta \lambda x^{\lambda-1}\right) e^{-\left(\theta x^{\alpha}+\beta x^{\lambda}\right)},
$$

Here $\theta$ and $\beta$ are scale parameters, and $\alpha$ and $\lambda$ are shape parameters. The interpretation of model (2) is evident. Suppose a system composed of two interconnected independent series sub-systems that affect the system in a different way, each one having a Weibull distribution with proper parameters. The hazard time of the system follows (2), since it occurs when the first of the two sub-systems fails. Let $X_{(1)}=\min \left(X_{i}\right)_{i=1}^{N}$, then the conditional cumulative distribution of $X_{(1)} \mid N=n$ is given by

$$
G_{\left\{X^{(1)} \mid N=n\right\}}(x)=1-[1-F(x)]^{\mathrm{n}}=1-e^{-n\left(\theta x^{\alpha}+\beta x^{\lambda}\right)},
$$

the cumulative distribution function of $\mathrm{X}_{(1)}$ is given by

$$
\begin{gathered}
F(x ; \theta, \alpha, \beta, \lambda, p)=(1-p) \sum_{n=1}^{\infty} p^{\mathrm{n}-1}\left[1-e^{-n\left(\theta x^{\alpha}+\beta x^{\lambda}\right)}\right] \\
=\frac{1-e^{-\left(\theta x^{\alpha}+\beta x^{\lambda}\right)}}{1-p e^{-\left(\theta x^{\alpha}+\beta x^{\lambda}\right)}}, \quad x \geq 0
\end{gathered}
$$

The corresponding probability density function $(p d f)$ of $X_{(1)}$ is given by

$$
f(x, \theta, \alpha, \beta, \lambda, p)=\frac{(1-p)\left(\theta \alpha x^{\alpha-1}+\beta \lambda x^{\lambda-1}\right) e^{-\left(\theta x^{\alpha}+\beta x^{\lambda}\right)}}{\left(1-p e^{-\left(\theta x^{\alpha}+\beta x^{\lambda}\right)}\right)^{2}}, x \geq 0
$$

We shall refer to the distribution given by Equations (4) and (5) as the as the additive Weibull geometric $(A W G)$ distribution. If a random variable $\mathrm{X}$ has the $A W G$ distribution, then we write $X \sim A W G(\theta, \alpha, \beta, \lambda, p)$.

The survival, hazard (hf) and reverse hazard (rh) functions of the (AWG) distribution are given respectively by

$$
\begin{aligned}
& \bar{F}(x ; \theta, \alpha, \beta, \lambda, p)=\frac{(1-p) e^{-\left(\theta x^{\alpha}+\beta x^{\lambda}\right)}}{1-p e^{-\left(\theta x^{\alpha}+\beta x^{\lambda}\right)}} \\
& h(x)=\frac{f(x)}{(1-F(x))}=\frac{\left(\theta \alpha x^{\alpha-1}+\beta \lambda x^{\lambda-1}\right)}{1-p e^{-\left(\theta x^{\alpha}+\beta x^{\lambda}\right)}},
\end{aligned}
$$

and

$$
\tau(x)=\frac{f(x)}{F(x)}=\frac{(1-p)\left(\theta \alpha x^{\alpha-1}+\beta \lambda x^{\lambda-1}\right) e^{-\left(\theta x^{\alpha}+\beta x^{\lambda}\right)}}{\left\{1-p e^{-\left(\theta x^{\alpha}+\beta x^{\lambda}\right)}\right\}\left\{1-e^{-\left(\theta x^{\alpha}+\beta x^{\lambda}\right)}\right\}} .
$$

respectively.

Figure 1 (a) and (b) provide some plots of the density curves for different values of the parameters $\theta, \alpha, \beta, \lambda$ and $p$ 


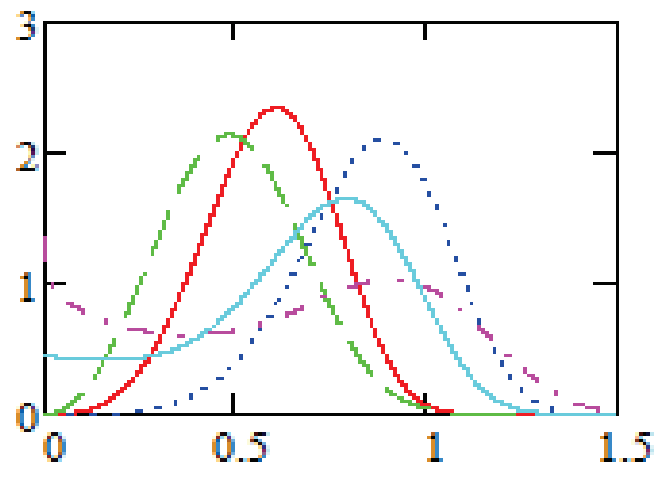

(a)

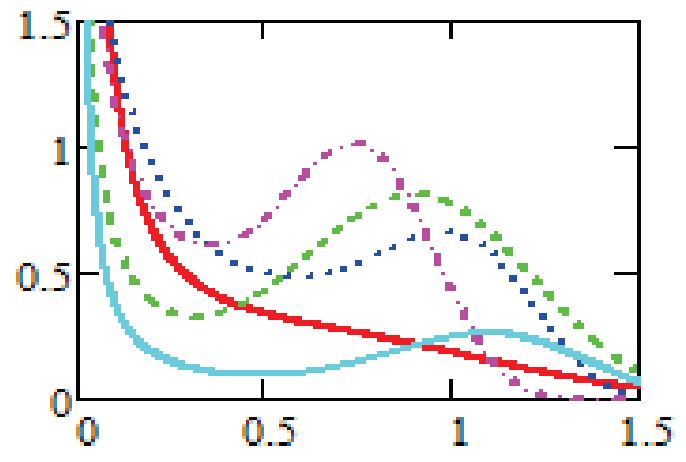

(b)

Fig.1. Plots of the $A W G$ density function for some parameter values. (a) For different values of $\theta, \alpha, \beta, \lambda$ with $p=0.4$.(b) For different values of $\theta, \alpha, \beta, \lambda$ with $p=0.1$.

Figure 2 does the same for the associated hazard rate function, showing that it is quite flexible for modelling survival data.

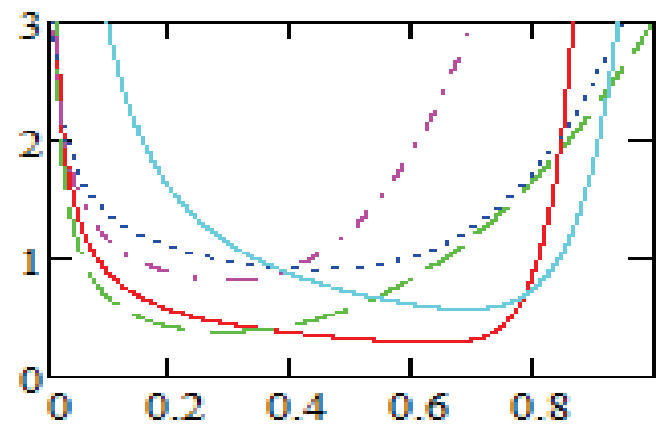

(a)

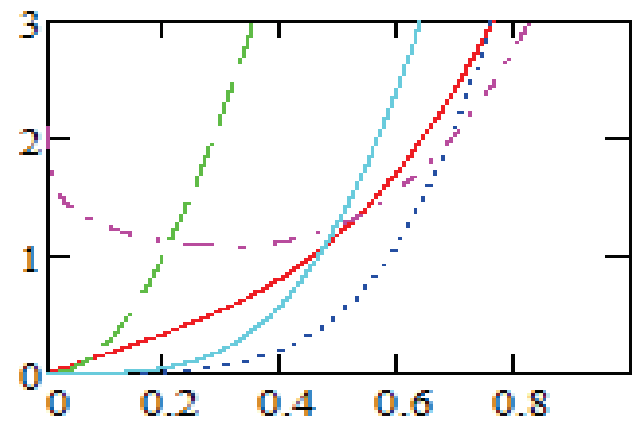

(b)

Fig. 2. (a) and (b) are plots of the hazard rate function for some parameter values. (a) For different values of $\theta, \alpha, \beta, \lambda$ with $p=0.4$.(b) For different values of $\theta, \alpha, \beta, \lambda$ with $p=0.1$.

It is known, not many lifetime distributions exhibit bathtub hazard rates. The $A W G$ model shows flexibility in accommodating all forms of the hazard rate function as seen from Figure (2) (by changing its parameter values) seems to be an important distribution that can be used.

\subsection{Special Cases of the AWG Distribution}

The exponentiated modified Weibull- geometric is very flexible model that approaches to different distributions when its parameters are changed. The flexibility of the exponentiated modified Weibullgeometric distribution is explained in the following. If $X$ is a random variable with cdf (4), then we have the following cases.

Special Cases:

1- If $=1$, then (4) reduces to the modified Weibull geometric which introduced by (Wang and Elbatal (2014)).

2- If $\theta=0$ we get the Weibull geometric distribution which introduced by (Souza et al. (2012)).

3- $\alpha=1$ and $\lambda=2$ we get the linear failure rate geometric distribution.

4- For $\alpha=1$ and $\beta=0$ we get the exponential geometric distribution. 
5- If $\theta=0$ and $\lambda=2$, we get the Rayleigh geometric distribution.

6- For $p \downarrow 0$ and $\alpha=1$ we get the modified Weibull distribution.

7- For $p \downarrow 0$ and $\theta=0$ we get the Weibull distribution.

8- For $\downarrow 0, \alpha=1$ and $\lambda=2$ we get the linear failure rate distribution.

9- For $\downarrow 0, \alpha=1$ and $\beta=0$ we get the exponential distribution.

10 - For $p \downarrow 0, \theta=0$ and $=2$, we get the Rayleigh distribution.

Note that, using the fact that if $|z|<1$ and $k>0$; we have the series representation

using (8) in equation (5) yields

$$
(1-z)^{-k}=\sum_{j=0}^{\infty} \frac{(\Gamma(k+j)}{\Gamma(k) j !} z^{j}
$$

$$
f(x ; \theta, \alpha, \beta, \lambda, p)=(1-p)\left(\theta \alpha x^{\alpha-1}+\beta \lambda x^{\lambda-1}\right) \sum_{j=0}^{\infty} p^{j}(j+1) e^{-(j+1)\left(\theta x^{\alpha}+\beta x^{\lambda}\right)}
$$

but the series expansion of $e^{-(j+1)\left(\beta x^{\lambda}\right)}$ is given by

$$
e^{-(j+1)\left(\beta x^{\lambda}\right)}=\sum_{k=0}^{\infty} \frac{\left[-\beta(j+1) x^{\lambda}\right]^{k}}{k !}
$$

substituting from (10) into (9), we get

$$
\begin{gathered}
f(x ; \theta, \alpha, \beta, \lambda, p)=(1-p) \sum_{j, K=0}^{\infty} \frac{p^{j}(-\beta)^{k}(j+1)^{k+1} x^{\lambda k}}{k !} e^{-(j+1)\left(\theta x^{\alpha}+\beta x^{\lambda}\right)} \\
\times\left(\theta \alpha x^{\alpha+\lambda k-1}+\beta \lambda x^{\lambda(k+1)-1}\right) e^{-(j+1)\left(\theta x^{\alpha}\right)} \\
=\omega_{(j, k)}\left(\theta \alpha x^{\alpha+\lambda k-1}+\beta \lambda x^{\lambda(k+1)-1}\right) e^{-(j+1)\left(\theta x^{\alpha}\right)},
\end{gathered}
$$

where

$$
\omega_{(j, k)}=(1-p) \sum_{j, K=0}^{\infty} \frac{p^{j}(-\beta)^{k}(j+1)^{k+1}}{k !}
$$

\section{Statistical Properties}

In this section we discuss the statistical properties of the additive Weibull geometric distribution, in particular, moment, moment generating function and conditional moment.

\subsection{Moments}

In this subsection we discuss the $r_{t h}$ moment for $A W G(\theta, \alpha, \beta, \lambda, p)$ distribution. Moments are necessary and important in any statistical analysis, especially in applications. It can be used to study the most important features and characteristics of a distribution (e.g., tendency, dispersion, skewness and kurtosis).

\section{Theorem 1.}

If $\mathrm{X}$ has $A W G(\phi, x), \phi=(\theta, \alpha, \beta, \lambda, p)$ then the $\mathrm{r}_{\mathrm{th}}$ moment of $\mathrm{X}$ is given by the following 
I. Elbatal et al.

$$
\dot{\mu}_{r}(x)=\omega_{(j, k)}\left[\frac{\theta \Gamma\left(\frac{r+\lambda k}{\alpha}+1\right)}{[(j+1) \theta]^{\frac{r+\lambda k+1}{\alpha}}}+\frac{\beta \lambda \Gamma\left(\frac{r+\lambda(k+1)}{\alpha}\right)}{\alpha[(j+1) \theta]^{\frac{r+\lambda(k+1)}{\alpha}}}\right]
$$

\section{Proof:}

Let $\mathrm{X}$ be a random variable with density function (11). The $\mathrm{r}_{\text {th }}$ ordinary moment of the $A W G$ distribution is given by

$$
\begin{gathered}
\dot{\mu}_{r}(x)=E\left(X^{r}\right)=\int_{0}^{\infty} x^{r} f(x, \phi) d x \\
=\int_{0}^{\infty} \omega_{(j, k)}\left(\theta \alpha x^{r+\alpha+\lambda k-1}+\beta \lambda x^{r+\lambda(k+1)-1}\right) e^{-(j+1)\left(\theta x^{\alpha}\right)} d x \\
=\omega_{(j, k)}\left[\frac{\theta \Gamma\left(\frac{r+\lambda k}{\alpha}+1\right)}{[(j+1) \theta]^{\frac{r+\lambda k+1}{\alpha}}}+\frac{\beta \lambda \Gamma\left(\frac{r+\lambda(k+1)}{\alpha}\right)}{\alpha[(j+1) \theta]^{\frac{r+\lambda(k+1)}{\alpha}}}\right] .
\end{gathered}
$$

Which completes the proof.

The central moments $\mu_{r}$ and cumulants $\kappa_{r}$ of the $A W G$ distribution can be determined from expression (12) as $\mu_{r}=\sum_{m=0}^{r}(-1)^{m} \mu_{1} \dot{\mu}_{r-m}$ and $\kappa_{r}=\dot{\mu}_{r-} \sum_{m=1}^{r-1}\left(\begin{array}{c}r-1 \\ m-1\end{array}\right) k_{m} \dot{\mu}_{r-m}$, respectively, Additionally, the skewness and kurtosis can be calculated from the third and fourth standardized cumulants in the forms $S K=$ $\left.\left(\left(\kappa_{3}\right) /\left(\sqrt{\left(\kappa_{2}\right.}{ }^{3}\right)\right)\right)$ and $K U=\left(\left(\kappa_{4}\right) /\left(\kappa_{2}{ }^{2}\right)\right)$, respectively.

\section{Theorem 2.}

The moment generating function of AWG distribution is given by

$$
M_{X}(t)=\sum_{r=0}^{\infty} \frac{t^{r}}{r !} \omega_{(j, k)}\left[\frac{\theta \Gamma\left(\frac{r+\lambda k}{\alpha}+1\right)}{[(j+1) \theta]^{\frac{r+\lambda k}{\alpha}+1}}+\frac{\beta \lambda \Gamma\left(\frac{r+\lambda(k+1)}{\alpha}\right)}{\alpha[(j+1) \theta]^{\frac{r+\lambda(k+1)}{\alpha}}}\right]
$$

\section{Proof.}

We start with the well-known definition of the moment generating function given by $M_{X}(t)=E\left(e^{t x}\right)=$ $\int_{0}^{\infty} e^{t x} f_{A W G}(x, \phi)$, since $\sum_{r=0}^{\infty} \frac{t^{r}}{r !} x^{r} f(x)$ converges and each term is integrable for all $\mathrm{t}$ close to 0 , then we can rewrite the moment generating function as $M_{X}(t)=\sum_{r=0}^{\infty} \frac{t^{r}}{r !} E\left(x^{r}\right)$ by replacing $E\left(x^{r}\right)$. Hence using (12) the $M G F$ of $A W G$ distribution is given by

$$
M_{X}(t)=\sum_{r=0}^{\infty} \frac{t^{r}}{r !} \omega_{(j, k)}\left[\frac{\theta \Gamma\left(\frac{r+\lambda k}{\alpha}+1\right)}{[(j+1) \theta]^{\frac{r+\lambda k}{\alpha}+1}}+\frac{\beta \lambda \Gamma\left(\frac{r+\lambda(k+1)}{\alpha}\right)}{\alpha[(j+1) \theta]^{\frac{r+\lambda(k+1)}{\alpha}}}\right] .
$$

which completes the proof.

Similarly, the characteristic function of the $A W G$ distribution becomes $\varphi_{X}(t)=M_{X}(i t)$ where $i=\sqrt{ }(-1)$ is the unit imaginary number. 


\subsection{Conditional Moments}

The main application of the first incomplete moment refers to the Bonferroni and Lorenz curves. These curves are very useful in economics, reliability, demography, insurance and medicine. The answers to many important questions in economics require more than just knowing the mean of the distribution, but its shape as well. This is obvious not only in the study of econometrics but in other areas as well. For lifetime models, it is also of interest to find the conditional moments and the mean residual lifetime function. The conditional moments for $A W G$ distribution is given by

$$
\begin{gathered}
v_{s}=E(X \mid X>t)-t=\int_{t}^{\infty} x^{s} f_{A W G}(x, \phi) d x \\
=\omega_{(j, k)} \int_{t}^{\infty}\left(\theta \alpha x^{s+\alpha+\lambda k-1}+\beta \lambda x^{s+\lambda(k+1)-1}\right) e^{-(j+1)\left(\theta x^{\alpha}\right)} d x \\
=\omega_{(j, k)}\left[\frac{\theta \Gamma\left(\frac{s+\lambda k}{\alpha}+1,(j+1) \theta t^{\alpha}\right)}{[(j+1) \theta]^{\frac{\lambda k+1}{\alpha}+1}}+\frac{\beta \lambda \Gamma\left(\frac{s+\lambda(k+1)+1}{\alpha},(j+1) \theta t^{\alpha}\right)}{\alpha[(j+1) \theta]^{\frac{s+\lambda(k+1)}{\alpha}}}\right] .
\end{gathered}
$$

Where $\Gamma(s, t)=\int_{t}^{\infty} x^{s-1} e^{-x} d x$ is the upper incomplete gamma function. The mean residual lifetime function is given by

$$
v_{s}=E(X \mid X>t)-t=\omega_{(j, k)}\left[\frac{\theta \Gamma\left(\frac{\lambda k}{\alpha}+1,(j+1) \theta t^{\alpha}\right)}{[(j+1) \theta]^{\frac{\lambda k+1}{\alpha}+1}}+\frac{\beta \lambda \Gamma\left(\frac{\lambda(k+1)+1}{\alpha},(j+1) \theta t^{\alpha}\right)}{\alpha[(j+1) \theta]^{\frac{\lambda(k+1)}{\alpha}}}\right]-t .
$$

The importance of the $M R L$ function is due to its uniquely determination of the lifetime distribution as well as the failure rate $(F R)$ function. Lifetimes can exhibit $I M R L$ (increasing $M R L$ ) or $D M R L$ (decreasing $M R L$ ). $M R L$ functions that first decreases (increases) and then increases (decreases) are usually called bathtub (upside-down bathtub) shaped, BMRL (UMRL). Many authors such as Ghitany (1998), Mi (1995), Park (1985) and Tang et al. (1999) have been studied the relationship between the behaviors of the $M R L$ and $F R$ functions of a distribution.

\section{Numbering and spacing}

Given that a component survives up to time $t \geq 0$, the residual life is the period beyond $t$ until the time of failure and defined by the conditional random variable $X-t \mid X>t$. In reliability, it is well known that the mean residual life function and ratio of two consecutive moments of residual life determine the distribution uniquely (Gupta and Gupta, 1983). Therefore, we obtain the $r^{\text {th }}$-order moment of the residual lifetime can be obtained via the general formula

$$
\mu_{r}(t)=E\left((X-t)^{r} \mid X>t\right)=\frac{1}{\bar{F}(t)} \int_{t}^{\infty}(x-t)^{r} f(x, \phi) d x, r \geq 1 .
$$

Applying the binomial expansion of $(x-t)^{r}$ into the above formula, we get

$$
\mu_{r}(t)=\frac{1}{\bar{F}(t)} \sum_{d=0}^{r}(-t)^{d}\left(\begin{array}{l}
r \\
d
\end{array}\right) \int_{t}^{\infty} x^{r-d} f(x) d x
$$




$$
\begin{array}{r}
=\frac{\omega_{(j, k)}}{\bar{F}(t)} \sum_{d=0}^{r}(-t)^{d}\left(\begin{array}{l}
r \\
d
\end{array}\right) \int_{t}^{\infty}\left(\theta \alpha x^{r+\alpha+\lambda k-d-1}+\beta \lambda x^{r+\lambda(k+1)-d-1}\right) e^{-(j+1)\left(\theta x^{\alpha}\right)} d x \\
=\frac{\omega_{(j, k)}}{\bar{F}(t)} \sum_{d=0}^{r}(-t)^{d}\left(\begin{array}{l}
r \\
d
\end{array}\right)\left[\frac{\theta \Gamma\left(\frac{\lambda k}{\alpha}+1,(j+1) \theta t^{\alpha}\right)}{[(j+1) \theta]^{\frac{\lambda k+1}{\alpha}+1}}+\frac{\beta \lambda \Gamma\left(\frac{\lambda(k+1)+1}{\alpha},(j+1) \theta t^{\alpha}\right)}{\alpha[(j+1) \theta]^{\frac{\lambda(k+1)}{\alpha}}}\right]
\end{array}
$$

The mean residual life $(M R L)$ of the $A W G$ distribution is given by

$$
\mu(t)=\frac{\omega_{(j, k)}}{\bar{F}(t)}\left[\frac{\theta \Gamma\left(\frac{\lambda k}{\alpha}+1,(j+1) \theta t^{\alpha}\right)}{[(j+1) \theta]^{\frac{\lambda k+1}{\alpha}+1}}+\frac{\beta \lambda \Gamma\left(\frac{\lambda(k+1)+1}{\alpha},(j+1) \theta t^{\alpha}\right)}{\alpha[(j+1) \theta]^{\frac{\lambda(k+1)}{\alpha}}}\right]-t
$$

The variance of the residual life of the $A W G$ distribution can be obtained easily by using $\mu_{2}(t)$ and $\mu(t)$.

On the other hand, we analogously discuss the reversed residual life and some of its properties. The reversed residual life can be defined as the conditional random variable $t-X \mid X \leq t$ which denotes the time elapsed from the failure of a component given that its life is less than or equal to t. This random variable may also be called the inactivity time (or time since failure); for more details, you may (see, Kundu and Nanda, (2010) and Nanda et al. (2003). Also, in reliability, the mean reversed residual life and ratio of two consecutive moments of reversed residual life characterize the distribution uniquely. the reversed failure (or reversed hazard) rate function is given by Equation (2.5). The $r^{\text {th }}$-order moment of the reversed residual life can be obtained by the well-known formula

$$
m_{r}(t)=E\left((t-X)^{r} \mid X \leq t\right)=\frac{1}{F(t)} \int_{0}^{t}(t-X)^{r} f(x, \phi) d x, r \geq 1 .
$$

Applying the binomial expansion of $(t-X)^{r}$ into the above formula gives

$$
\begin{gathered}
m_{r}(t)=\frac{\omega_{(i, j, k)}}{F(t)} \sum_{d=0}^{r}(-t)^{d}\left(\begin{array}{l}
r \\
d
\end{array}\right) \int_{0}^{t}\left(\theta \alpha x^{r+\alpha+\lambda k-d-1}+\beta \lambda x^{r+\lambda(k+1)-d-1}\right) e^{-(j+1)\left(\theta x^{\alpha}\right)} d x \\
=\frac{\omega_{(i, j, k)}}{F(t)} \sum_{d=0}^{r}(-t)^{d}\left(\begin{array}{l}
r \\
d
\end{array}\right)\left[\frac{\theta \zeta\left(\frac{r+\lambda k-d}{\alpha}+1,(j+1) \theta t^{\alpha}\right)}{[(j+1) \theta]^{\frac{r+\lambda k-d}{\alpha}+1}}+\frac{\beta \lambda \Gamma\left(\frac{r+\lambda(k+1)-d}{\alpha},(j+1) \theta t^{\alpha}\right)}{\alpha[(j+1) \theta]^{\frac{r+\lambda(k+1)-d}{\alpha}}}\right]
\end{gathered}
$$

where $\zeta(s, t)=\int_{0}^{t} x^{s-1} e^{-x} d x$ is the lower incomplete gamma function. Thus, the mean of the reversed residual life of the AWG distribution is given by

$$
m(t)=t-\frac{\omega_{(j, k)}}{F(t)}\left[\frac{\theta \Gamma\left(\frac{\lambda k}{\alpha}+1,(j+1) \theta t^{\alpha}\right)}{[(j+1) \theta]^{\frac{\lambda k+1}{\alpha}+1}}+\frac{\beta \lambda \Gamma\left(\frac{\lambda(k+1)+1}{\alpha},(j+1) \theta t^{\alpha}\right)}{\alpha[(j+1) \theta]^{\frac{\lambda(k+1)}{\alpha}}}\right]
$$

Using $m(t)$ and $m_{2}(t)$ one can obtain the variance and the coefficient of variation of the reversed residual life of the $A W G$ distribution. 


\subsection{Bonferroni and Lorenz Curves}

In this subsection we proposed the Bonferroni and Lorenz Curves. The Bonferroni and Lorenz curves (Bonferroni 1930) and the Bonferroni and Gini indices have applications not only in economics to study income and poverty, but also in other fields like reliability, demography, insurance and medicine. The Bonferroni and Lorenz curves are defined by

$$
\begin{aligned}
B(p) & =\frac{1}{p \mu} \int_{0}^{q} x f(x) d x \\
& =\frac{\omega_{(j, k)}}{p \mu}\left[\frac{\theta \zeta \Gamma\left(\frac{\lambda k+1}{\alpha}+1,(j+1) \theta q^{\alpha}\right)}{[(j+1) \theta]^{\frac{\lambda k+1}{\alpha}+1}}+\frac{\beta \lambda \zeta \Gamma\left(\frac{\lambda(k+1)+1}{\alpha},(j+1) \theta q^{\alpha}\right)}{\alpha[(j+1) \theta]^{\frac{\lambda(k+1)+1}{\alpha}}}\right]
\end{aligned}
$$

and

$$
\begin{aligned}
L(p) & =\frac{1}{\mu} \int_{0}^{q} x f(x) d x \\
& =\frac{\omega_{(j, k)}}{\mu}\left[\frac{\theta \zeta \Gamma\left(\frac{\lambda k+1}{\alpha}+1,(j+1) \theta q^{\alpha}\right)}{[(j+1) \theta]^{\frac{\lambda k+1}{\alpha}+1}}+\frac{\beta \lambda \zeta \Gamma\left(\frac{\lambda(k+1)+1}{\alpha},(j+1) \theta q^{\alpha}\right)}{\alpha[(j+1) \theta]^{\frac{\lambda(k+1)+1}{\alpha}}}\right]
\end{aligned}
$$

\subsection{Mean deviation}

In statistics, mean deviation about the mean and mean deviation about the median measure the ammount of scatter in a population. For random variable $X$ with pdf $(x)$, distribution function $F(x)$, mean $\mu=E(X)$ and $M=$ Median $(X)$, the mean deviation about the mean and mean deviation about the median, are defined by

$$
\begin{aligned}
\delta_{1}(x)=\int_{0}^{\infty}|x-\mu| f(x) & d x \\
& =2 \mu F(\mu)-2 \mu+2 \int_{\mu}^{\infty} x f(x) d x .
\end{aligned}
$$

and

$$
\begin{aligned}
\delta_{2}(x)=\int_{0}^{\infty}|x-M| & f(x) d x \\
& =2 M F(M)-M-\mu+2 \int_{M}^{\infty} x f(x) d x .
\end{aligned}
$$

respectively, if $X$ is $A W G$ random variable then

$$
\int_{\mu}^{\infty} x f(x) d x=\omega_{(j, k)}\left[\frac{\theta \Gamma\left(\frac{\lambda k+1}{\alpha}+1,(j+1) \theta \mu^{\alpha}\right)}{[(j+1) \theta]^{\frac{\lambda k+1}{\alpha}+1}}+\frac{\beta \lambda \Gamma\left(\frac{\lambda(k+1)+1}{\alpha},(j+1) \theta \mu^{\alpha}\right)}{\alpha[(j+1) \theta]^{\frac{\lambda(k+1)+1}{\alpha}}}\right],
$$

and 


$$
\int_{M}^{\infty} x f(x) d x=\omega_{(j, k)}\left[\frac{\theta \Gamma\left(\frac{\lambda k+1}{\alpha}+1,(j+1) \theta M^{\alpha}\right)}{[(j+1) \theta]^{\frac{\lambda k+1}{\alpha}+1}}+\frac{\beta \lambda \Gamma\left(\frac{\lambda(k+1)+1}{\alpha},(j+1) \theta M^{\alpha}\right)}{\alpha[(j+1) \theta]^{\frac{\lambda(k+1)+1}{\alpha}}}\right],
$$

so that

$$
\delta^{1}(x)=2 \mu F(\mu)-2 \mu+2 \omega_{(j, k)}\left[\frac{\theta \Gamma\left(\frac{\lambda k+1}{\alpha}+1,(j+1) \theta \mu^{\alpha}\right)}{[(j+1) \theta]^{\frac{\lambda k+1}{\alpha}+1}}+\frac{\beta \lambda \Gamma\left(\frac{\lambda(k+1)+1}{\alpha},(j+1) \theta \mu^{\alpha}\right)}{\alpha[(j+1) \theta]^{\frac{\lambda(k+1)+1}{\alpha}}}\right],
$$

and

$$
\delta_{2}(x)=-\mu+2 \omega_{(j, k)}\left[\frac{\theta \Gamma\left(\frac{\lambda k+1}{\alpha}+1,(j+1) \theta M^{\alpha}\right)}{[(j+1) \theta]^{\frac{\lambda k+1}{\alpha}+1}}+\frac{\beta \lambda \Gamma\left(\frac{\lambda(k+1)+1}{\alpha},(j+1) \theta M^{\alpha}\right)}{\alpha[(j+1) \theta]^{\frac{\lambda(k+1)+1}{\alpha}}}\right] \text {. }
$$

\section{Maximum Likelihood Estimation}

Statistical inference can be carried out in three different ways: point estimation, interval estimation and hypothesis testing. Several approaches for parameter point estimation were proposed in the literature but the maximum likelihood method is the most commonly employed. The MLEs enjoy desirable properties and can be used when constructing confidence intervals and regions and also in test statistics. Large sample theory for these estimates delivers simple approximations that work well in finite samples. Statisticians often seek to approximate quantities such as the density of a test statistic that depend on the sample size in order to obtain better approximate distributions. The resulting approximation for the MLES in distribution theory is easily handled either analytically or numerically. Here, we determine the maximum likelihood estimates (MLEs) of the parameters of the $A W G$ distribution from complete samples only. Let $x_{1}, \ldots, x_{n}$ be a random sample of size $\mathrm{n}$ from the $A W G$ distribution given by (5). Let $\varphi=(\theta, \alpha, \beta, \lambda, p)^{T}$ be $p \times 1$ vector of parameters. The total $\log$-likelihood function for $\varphi$ is given by

$$
\begin{gathered}
L_{n}=L_{n}(\varnothing)=n \log (1-p)+\sum_{i=1}^{n} \log \left(\theta \alpha x_{i}^{\alpha-1}+\beta \lambda x_{i}^{\lambda-1}\right)-\theta \sum_{i=1}^{n} x_{i}{ }^{\alpha}-\beta \sum_{i=1}^{n} x_{i}{ }^{\lambda} \\
-2 \sum_{i=1}^{n} \log \left(1-p e^{-\left(\theta x_{i}{ }^{\alpha}+\beta x_{i}{ }^{\lambda}\right)}\right) .
\end{gathered}
$$

The log-likelihood can be maximized either directly by using the SAS program or R-language (2012) or by solving the nonlinear likelihood equations obtained by differentiating (23). The associated components of the score function

$$
\begin{aligned}
& U_{n}(\Phi)=\left[\frac{\partial L_{n}}{\partial \theta}, \frac{\partial L_{n}}{\partial \alpha}, \frac{\partial L_{n}}{\partial \beta}, \frac{\partial L_{n}}{\partial \lambda}, \frac{\partial L_{n}}{\partial p}\right]^{T} \text { are } \\
& \frac{\partial L_{n}}{\partial \theta}=\sum_{i=1}^{n} \frac{\alpha x_{i}^{\alpha-1}}{\left(\theta \alpha x_{i}^{\alpha-1}+\beta \lambda x_{i}^{\lambda-1}\right)}-\sum_{i=1}^{n} x_{i}^{\alpha}-2 p \sum_{i=1}^{n} \frac{x_{i}{ }^{\alpha} e^{-\left(\theta x_{i}^{\alpha}+\beta x_{i}{ }^{\lambda}\right)}}{\left(1-p e^{-\left(\theta x_{i}^{\alpha}+\beta x_{i}^{\lambda}\right)}\right)^{\prime}} \\
& \frac{\partial L_{n}}{\partial \alpha}=\sum_{i=1}^{n} \frac{\theta x_{i}{ }^{\alpha-1}\left(\alpha \ln \left(x_{i}\right)+1\right)}{\left(\theta \alpha x_{i}{ }^{\alpha-1}+\beta \lambda x_{i}^{\lambda-1}\right)}-\theta \sum_{i=1}^{n} x_{i}{ }^{\alpha} \ln \left(x_{i}\right)-2 p \sum_{i=1}^{n} \frac{\theta x_{i}{ }^{\alpha} \ln \left(x_{i}\right) e^{-\left(\theta x_{i}{ }^{\alpha}+\beta x_{i}{ }^{\lambda}\right)}}{\left(1-p e^{-\left(\theta x_{i}{ }^{\alpha}+\beta x_{i}{ }^{\lambda}\right)}\right)} \text {, } \\
& \frac{\partial L_{n}}{\partial \beta}=\sum_{i=1}^{n} \frac{\lambda x_{i}^{\lambda-1}}{\left(\theta \alpha x_{i}^{\alpha-1}+\beta \lambda x_{i}^{\lambda-1}\right)}-\sum_{i=1}^{n} x_{i}^{\lambda}-2 p \sum_{i=1}^{n} \frac{x_{i}^{\lambda} e^{-\left(\theta x_{i}{ }^{\alpha}+\beta x_{i}{ }^{\lambda}\right)}}{\left(1-p e^{-\left(\theta x_{i}{ }^{\alpha}+\beta x_{i}{ }^{\lambda}\right)}\right)^{\prime}} \\
& \frac{\partial L_{n}}{\partial \lambda}=\sum_{i=1}^{n} \frac{\beta x_{i}^{\lambda-1}\left(\alpha \ln \left(x_{i}\right)+1\right)}{\left(\theta \alpha x_{i}{ }^{\alpha-1}+\beta \lambda x_{i}^{\lambda-1}\right)}-\beta \sum_{i=1}^{n} x_{i}^{\lambda} \ln \left(x_{i}\right)-2 p \sum_{i=1}^{n} \frac{\beta x_{i}^{\lambda} \ln \left(x_{i}\right) e^{-\left(\theta x_{i}{ }^{\alpha}+\beta x_{i}{ }^{\lambda}\right)}}{\left(1-p e^{-\left(\theta x_{i}{ }^{\alpha}+\beta x_{i}{ }^{\lambda}\right)}\right)} \text {, }
\end{aligned}
$$


and

$$
\frac{\partial L_{n}}{\partial p}=\frac{-n}{1-p}+2 \sum_{i=1}^{n} \frac{e^{-\left(\theta x_{i}{ }^{\alpha}+\beta x_{i}{ }^{\lambda}\right)}}{\left(1-p e^{-\left(\theta x_{i}{ }^{\alpha}+\beta x_{i}{ }^{\lambda}\right)}\right)} .
$$

And The maximum likelihood estimation (MLE) of $\phi$, say $\hat{\phi}$, is obtained by solving the nonlinear system $U_{n}(\phi)=0$. These equations cannot be solved analytically, and statistical software can be used to solve them numerically via iterative methods. We can use iterative techniques such as a Newton--Raphson type algorithm to obtain the estimate $\hat{\phi}$. For interval estimation and hypothesis tests on the model parameters, we require the information matrix. The $5 \times 5$ observed information matrix is given by

$$
I_{n}(\varphi)=-\left(\begin{array}{lllll}
I_{\theta \theta} & I_{\theta \alpha} & I_{\theta \beta} & I_{\theta \lambda} & I_{\theta p} \\
I_{\alpha \theta} & I_{\alpha \alpha} & I_{\alpha \beta} & I_{\alpha \lambda} & I_{\alpha p} \\
I_{\beta \beta} & I_{\beta \alpha} & I_{\beta \beta} & I_{\beta \lambda} & I_{\beta p} \\
I_{\lambda \theta} & I_{\lambda \alpha} & I_{\lambda \beta} & I_{\lambda \lambda} & I_{\lambda p} \\
I_{p \theta} & I_{p \alpha} & I_{p \beta} & I_{p \lambda} & I_{p p}
\end{array}\right)
$$

whose elements are given in Appendix. Applying the usual large sample approximation, $M L E$ of $\phi$, i.e $\hat{\phi}$ can be treated as being approximately $N_{5}\left(\phi, J_{n}(\phi)^{-1}\right)$, where $J_{n}(\phi)=E\left[I_{n}(\phi)\right]$. Under conditions that are fulfilled for parameters in the interior of the parameter space but not on the boundary, the asymptotic distribution of $\sqrt{n}(\hat{\phi}-\phi)$ is ${ }_{5}\left(0, J(\phi)^{-1}\right)$, where $J(\phi)=\lim _{-}\{n \rightarrow \infty\} n^{-1} I_{n}(\phi)$ is the unit information matrix. This asymptotic behavior remains valid if $J(\phi)$ is replaced by the average sample information matrix evaluated at $\hat{\phi}$, say $n^{-1} I_{n}(\hat{\phi})$. The estimated asymptotic multivariate normal $N_{5}\left(\phi, I_{n}(\hat{\phi})^{-1}\right)$ distribution of $\phi$ can be used to construct approximate confidence intervals for the parameters and for the hazard rate and survival functions. An 100(1- $\gamma$ ) asymptotic confidence interval for each parameter $\phi_{r}$ is given by

$$
A C I_{r}=\left(\phi_{r}-z_{\frac{\gamma}{2}} \sqrt{\hat{I}_{r r}} \hat{\phi}_{r}+z_{\frac{\gamma}{2}} \sqrt{\hat{I}_{r r}}\right)
$$

where $z_{\gamma}$ is the upper $100 \gamma$ the percentile of the standard normal distribution.

\section{Applications}

In this section, we use two real data sets to see how the new model works in practice. compare the fits of the $A W G$ distribution with others models. In each case, the parameters are estimated by maximum likelihood as described in Section 5, using the R code.

\subsection{Data Set 1}

The first data set represents failure times are reported in the book Weibull Models by Murthy" et al. (2004, page 297) as follows:

$0.040,1.866,2.385,3.443,0.301,1.876,2.481,3.467,0.309,1.899,2.610,3.478,0.557,1.911,2.625$, $3.578,0.943,1.912,2.632,3.595,1.070,1.914,2.646,3.699,1.124,1.981,2.661,3.779,1.248,2.010,2.688$, $3.924,1.281,2.038,2.82,3,4.035,1.281,2.085,2.890,4.121,1.303,2.089,2.902,4.167,1.432,2.097,2.934$, $4.240,1.480,2.135,2.962,4.255,1.505,2.154,2.964,4.278,1.506,2.190,3.000,4.305,1.568,2.194,3.103$, $4.376,1.615,2.223,3.114,4.449,1.619,2.224,3.117,4.485,1.652,2.229,3.166,4.570,1.652,2.300,3.344$, $4.602,1.757,2.324,3.376,4.663$.

In order to compare the two distribution models, we consider criteria like KS (Kolmogorov Smirnov), -2L, AIC (Akaike information criterion), AICC (corrected Akaike information criterion), and BIC (Bayesian information criterion) for the data set. The better distribution corresponds to smaller KS, -2L, AIC and AICC values: 
I. Elbatal et al.

$$
\begin{gathered}
A I C=-2 L+2 K, \\
A I C C=-2 L+\left(\frac{2 K n}{n-K-1}\right),
\end{gathered}
$$

and

$$
B I C=-2 L+K \log (n)
$$

where $\mathrm{L}$ denotes the log-likelihood function evaluated at the maximum likelihood estimates, $\mathrm{K}$ is the number of parameters, and $\mathrm{n}$ is the sample size.

Also, for calculating the values of $K S$ we use the sample estimates of $\theta, \alpha, \beta, \lambda$ and $P$. Table 1 shows the parameter estimation based on the maximum likelihood and gives the values of the criteria $A I C, A I C C, B I C$, and $K S$ test. The values in Table 1 indicate that the $A W G$ distribution leads to a better fit over all the other

\begin{tabular}{|c|c|c|c|c|c|c|c|}
\hline Model & Estimates & $S E$ & $-\log L$ & $A I C$ & $A I C C$ & $B I C$ & $K S$ \\
\hline \multirow[t]{5}{*}{$A W G$} & $\hat{\theta}=0.011$ & 0.0001 & 127.11 & 264.22 & 264.98 & 264.98 & 0.07 \\
\hline & $\hat{\alpha}=3.483$ & 1.0215 & & & & & \\
\hline & $\hat{\beta}=0.019$ & 0.3341 & & & & & \\
\hline & $\hat{\lambda}=0.718$ & 0.3383 & & & & & \\
\hline & $\hat{p}=0.602$ & 0.0123 & & & & & \\
\hline \multirow[t]{5}{*}{$E M W G$} & $\hat{\theta}=18.15$ & 6.3254 & 129.25 & 268.50 & 269.26 & 269.26 & 0.08 \\
\hline & $\hat{\alpha}=1.312$ & 0.3254 & & & & & \\
\hline & $\hat{\beta}=0.110$ & 0.2446 & & & & & \\
\hline & $\hat{\lambda}=3.374$ & 1.0225 & & & & & \\
\hline & $\hat{p}=0.984$ & 0.0221 & & & & & \\
\hline \multirow[t]{4}{*}{$E W G$} & $\beta=0.593$ & 0.9991 & 129.28 & 266.56 & 267.06 & 276.33 & 0.82 \\
\hline & $\hat{\theta}=0.975$ & 0.3938 & & & & & \\
\hline & $\hat{\lambda}=1.545$ & 1.2363 & & & & & \\
\hline & $\hat{p}=0.986$ & 0.0142 & & & & & \\
\hline \multirow[t]{3}{*}{$W G$} & $\hat{\beta}=1.074$ & 0.4168 & 129.37 & 264.75 & 265.04 & 272.07 & 0.81 \\
\hline & $\hat{\theta}=1.232$ & 1.0650 & & & & & \\
\hline & $\hat{p}=0.962$ & 0.060 & & & & & \\
\hline \multirow[t]{2}{*}{$W$} & $\hat{\beta}=2.393$ & 0.210 & 131.28 & 266.57 & 266.72 & 271.46 & 0.09 \\
\hline & $\hat{\theta}=0.3486$ & 0.016 & & & & & \\
\hline$E$ & $\hat{\theta}=0.390$ & 0.0017 & 164.98 & 331.97 & 332.02 & 334.41 & 0.291 \\
\hline
\end{tabular}
models.

Table1.MLEs the measures AIC, AICC and BIC, and KS test. 


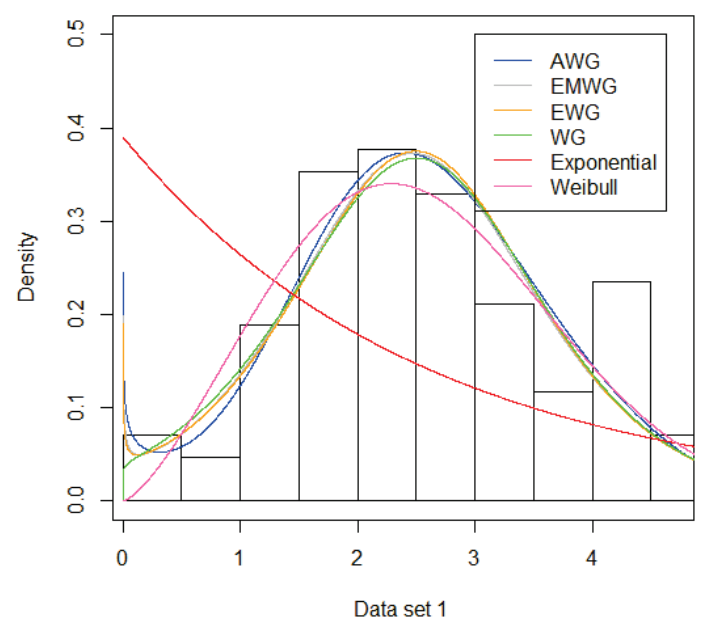

Fig. 3. Estimated densities of data set 1

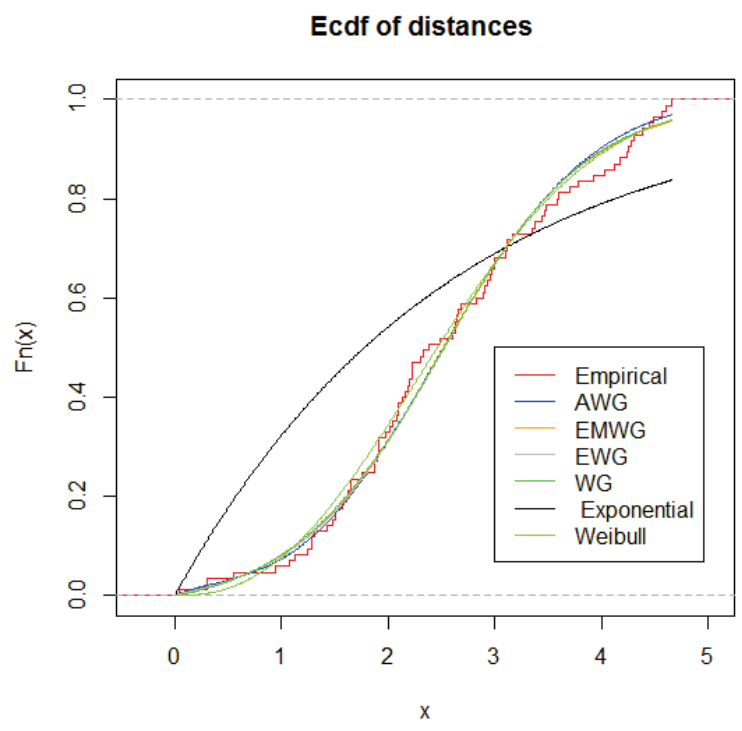

Fig. 4. Empirical, fitted AWG, EMWG, MWG, WG, Exponential and Weibull distributions of data set 1 . 

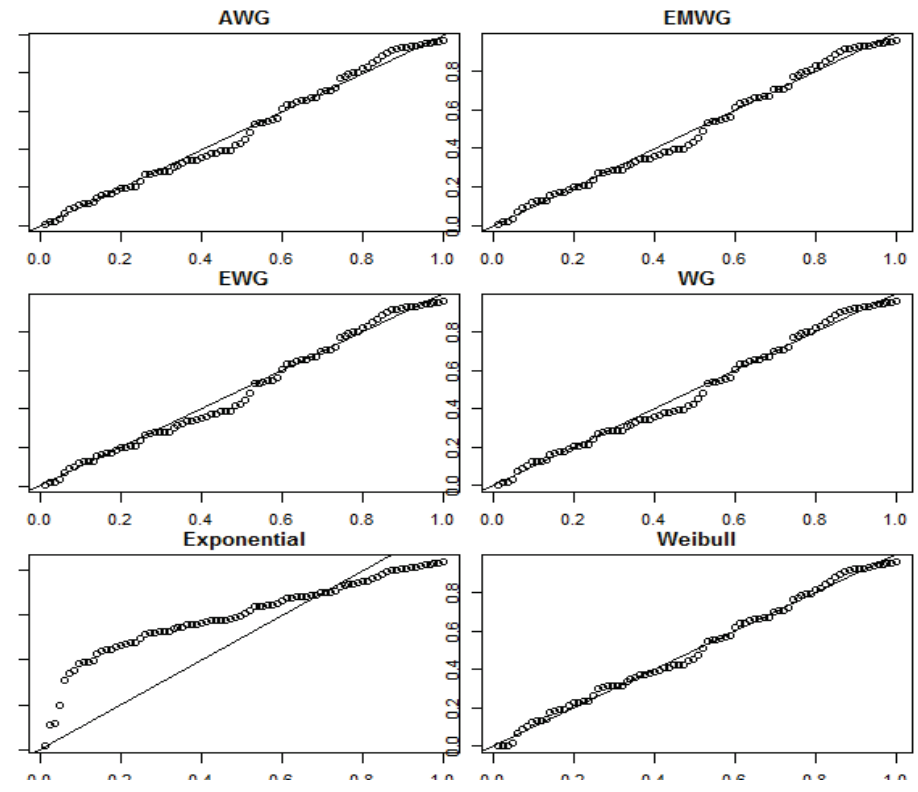

Fig. 5. Probability plots for the fits of the AWG, EMWG, MWG, WG, Exponential and Weibull distributions of data set1.

\subsection{Data Set 2}

The second data set represents failure time of 50 items reported in Aarset [1].

Table 2. MLEs the measures AIC, AICC and BIC, and KS test to failure time data for the models.

\begin{tabular}{|c|c|c|c|c|c|c|c|}
\hline Model & Estimates & $S E$ & $-\log L$ & $A I C$ & $A I C C$ & $B I C$ & $K S$ \\
\hline \multirow{5}{*}{$A W G$} & $\hat{\theta}=0.0001$ & 0.0000 & 229.39 & 468.78 & 470.14 & 478.34 & 0.13 \\
\hline & $\hat{\alpha}=2.1455$ & 1.0002 & & & & & \\
\hline & $\hat{\beta}=0.0825$ & 0.0496 & & & & & \\
\hline & $\hat{\lambda}=0.4178$ & 0.1087 & & & & & \\
\hline & $\hat{p}=0.0099$ & 0.4644 & & & & & \\
\hline \multirow[t]{5}{*}{$E M W G$} & $\hat{\theta}=13.569$ & 0.8470 & 233.51 & 477.03 & 478.40 & 486.59 & 0.15 \\
\hline & $\hat{\alpha}=0.0327$ & 0.0064 & & & & & \\
\hline & $\hat{\beta}=0.0452$ & 0.0377 & & & & & \\
\hline & $\hat{\lambda}=7.0182$ & 6.1686 & & & & & \\
\hline & $\hat{p}=0.9991$ & 0.0003 & & & & & \\
\hline \multirow[t]{4}{*}{$E W G$} & $\hat{\beta}=1.0111$ & 0.6691 & 234.83 & 477.66 & 478.55 & 485.31 & 0.14 \\
\hline & $\hat{\theta}=0.0259$ & 0.0038 & & & & & \\
\hline & $\hat{\lambda}=1.0689$ & 0.2463 & & & & & \\
\hline & $\hat{p}=0.0365$ & 0.0142 & & & & & \\
\hline \multirow[t]{3}{*}{$W G$} & $\hat{\beta}=0.699$ & 0.1582 & 237.70 & 481.41 & 481.93 & 487.15 & 0.16 \\
\hline & $\hat{\theta}=0.1585$ & 0.1380 & & & & & \\
\hline & $\hat{p}=0.8505$ & 0.1224 & & & & & \\
\hline \multirow[t]{2}{*}{$W$} & $\hat{\beta}=0.948$ & 0.1197 & 240.97 & 485.95 & 486.21 & 489.78 & 0.17 \\
\hline & $\hat{\theta}=0.0222$ & 0.0034 & & & & & \\
\hline$E$ & $\hat{\theta}=0.0218$ & 0.0000 & 241.06 & 484.13 & 484.21 & 486.04 & 0.64 \\
\hline
\end{tabular}




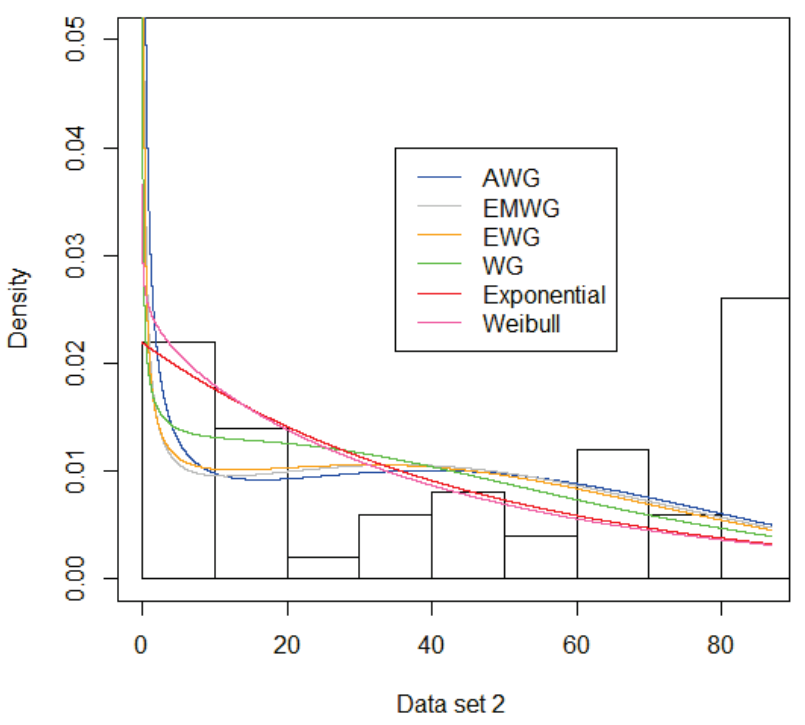

Fig 6. Estimated densities of data set 2.

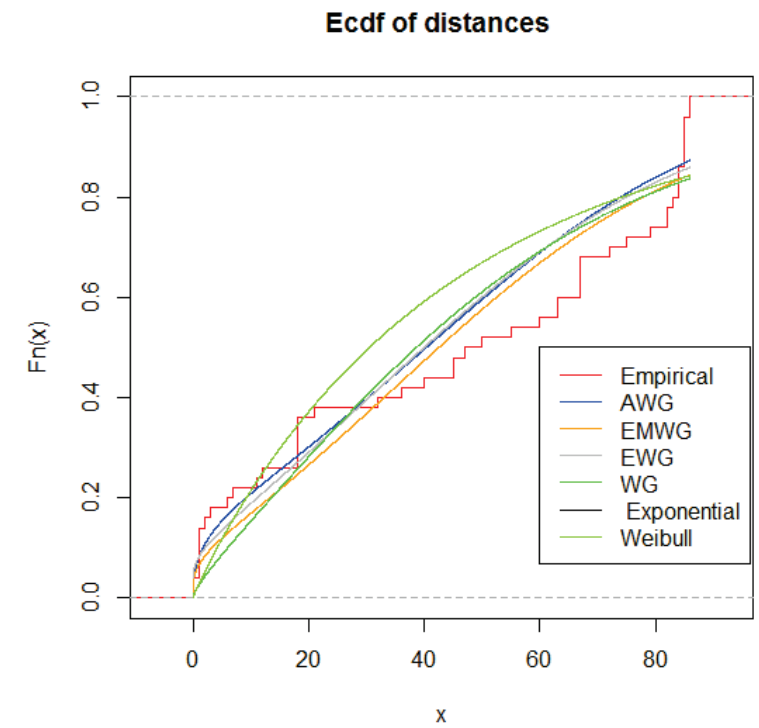

Fig 7. Empirical, fitted AWG, EMWG, MWG, WG,

Exponential and Weibull distributions of data set 2 . 

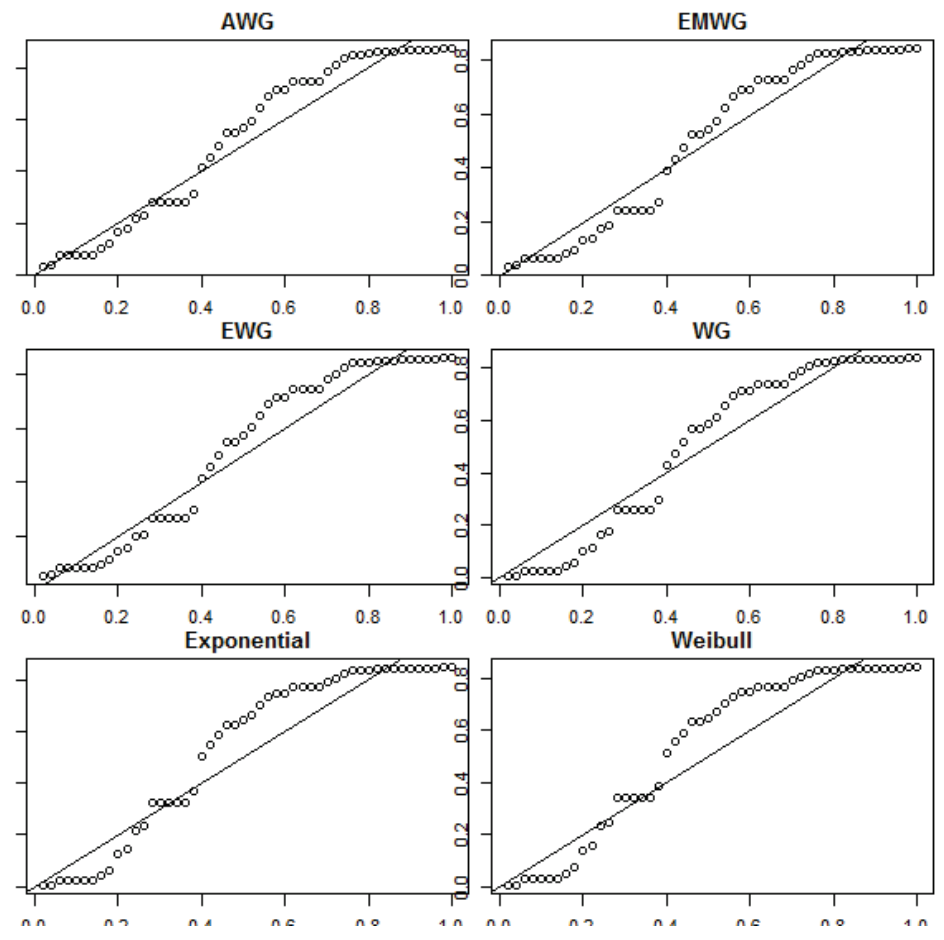

Fig 8. Probability plots for the fits of the AWG, EMWG, MWG, WG,

Exponential and Weibull distributions of data set2.

\section{References}

[1] M. V, Aarset, How to identify a bathtub hazard rate. IEEE Transactions on Reliability, 36(1), (1987)., 106-108.

[2] K. Adamidis, T. Dimitrakopoulou, and S. Loukas, On a generalization of the exponential-geometric distribution. Statistics \& Probability Letters, 73, (2005), pp 259-269.

[3] K. Adamidis K. and S. Loukas, A lifetime distribution with decreasing failure rate. Statistics \& Probability Letters, 39, (1998), pp 35- 42.

[4] W. Barreto-Souza, R.B. Silva and G. M. Cordeiro, A new distribution with decreasing, increasing and upside-down bathtub failure rate, Comput. Statist. Data Anal. 54, (2010), pp. 935-- 944.

[5] W. Barreto-Souza, A.L. de Morais, and G. M. Cordeiro, The Weibull-geometric distribution, J. Stat. Comput. Simul. 81, (2011), pp. 645-657.

[6] W. Barreto-Souza, and F. Cribari-Neto, A generalization of the exponential-Poisson distribution. Statistics \& Probability Letters, 79, (2009), pp 2493- 2500.

[7] C.E. Bonferroni, Elmenti di statistica generale. Libreria Seber, Firenze. (1930)

[8] M. Chahkandi, and M. Ganjali, On some lifetime distributions with decreasing failure rate. Computational Statistics \& Data Analysis, 53, (2009), pp 4433- 4440.

[9] N.L. Johnson, S. Kotz, and N. Balakrishnan, Continuous Univariate Distributions. John Wiley \& Sons, New York. (1994)

[10] J.F. Kenney, and E.S. Keeping, Mathematics of statistics, Part 1, 3rded. Princeton, NJ, (1962) pp. 101-102.

[11] C. Kundu, and A.K. Nanda, Some reliability properties of the inactivity time. Communications in Statistics Theory and Methods, 39, (2010), pp 899- 911.

[12] C. Kus, A new lifetime distribution. Computational Statistics \& Data Analysis, 51, (2007), pp 4497- 4509.

[13] C. D. Lai, M. Xie, and D. N. P. Murthy, A modified Weibull distribution. Transactions on Reliability, 52, (2003), pp 33-37.

[14] W. Lu, and D. Shi, A new compounding life distribution: The Weibull-Poisson distribution. Journal of Applied Statistics, (2011) DOI:10.1080/02664763.2011.575126.

[15] E. Mahmoudi and Shiran, introduced the exponentiated Weibull geometric distribution. arXiv:1206.4008v1 [stat.ME]. (2012). 
[16] A.L. Morais, and W. Barreto-Souza, A Compound Class of Weibull and Power Series Distributions. Computational Statistics \& Data Analysis, 55, (2011), pp 1410-1425.

[17] J.J.A Moors, A quantile alternative for kurtosis. Journal of the Royal Statistical Society Ser. D., The Statistician, 37, (1998), pp 25-32.

[18] DNP. Murthy, M. Xi, and R. Jiangs, Weibull models, Wiley, Hoboken, (2004).

[19] S. Nadarajah, F. Shahsanaei, and S. Sadegh Rezaei, A new four-parameter lifetime distribution. Journal of Statistical Computation and Simulation, (84)2, (2014), pp 248--263.

[20] A.K. Nanda, H. Singh, N. Misra, and P. Paul, Reliability properties of reversed residual lifetime. Communications in Statistics Theory and Methods, 32, (2003), pp2031- 2042.

[21] K. S. Park,Effect of burn-in on mean residual life, IEEE Transactions on Reliability. 34, (1985), pp 522-523.

[22] S. Rezaei, S.Nadarajah,and N. Tahghighnia, A new three-parameter lifetime distribution. Statistics 0, (2011), pp 1--26.

[23] A. M. Sarhan, and M. Zain-din, Modified Weibull distribution, Applied Sciences, (11), (2009), pp 123-136.

[24] J, S. Swain.S, Venkatraman. and J. Wilson, Least squares estimation of distribution function in Johnson's translation system. Journal of Statistical Computation and Simulation, 29, (1988), pp 271 - 297.

[25] R, Tahmasbi, and S. Rezaei, A two-parameter lifetime distribution with decreasing failure rate. Computational Statistics. \& Data Analysis, 52, (2008), pp 3889- 3901.

[26] L.C. Tang, Y. Lu, and E.P. Chew, Mean residual life distributions, IEEE Transactions on Reliability 48, (1999), pp 68-73.

[27] M. Wang and I. Elbatal, he modified Weibull geometric distribution METRON (In press) (2014)

[28] M. Xie, and C.D. Lai, Reliability analysis using an additive Weibull model with bath tub shaped failure rate function. Reliability Engineering and system safety,52. (1995), pp 87-93.

[29] H. Zakerzadeh, and E. Mahmoudi, A new two parameter lifetime distribution: model and properties. arXiv: 1204.4248 [stat. CO]. (2012). 